\title{
Cryopreservation of spermatozoa from cynomolgus monkeys (Macaca fascicularis)*
}

\author{
T. L. Tollner†, C. A. VandeVoort $\uparrow$, J. W. Overstreet $\dagger \ddagger$ and E. Z. Drobnis \\ $\uparrow$ California Primate Research Center; $¥$ Division of Reproductive Biology, Department of Obstetrics \\ and Gynecology, School of Medicine; and \$Department of Zoology, University of California, Davis, \\ California $95616, U S A$
}

\begin{abstract}
Summary. Three egg-yolk diluents, which have been used successfully in cryopreservation of human spermatozoa, were compared for their ability to protect macaque semen against cryodamage. TEST (Tes + Tris + egg yolk), TEST with $20 \%$ skim milk (TSM), and egg yolk-citrate (EYC), each with 3 or $5 \%$ glycerol were compared using 12 ejaculates from 6 male cynomolgus macaques. Computer-aided analysis of sperm motion was used to determine the percentage motility $(\% \mathrm{M})$, curvilinear velocity (VCL), and linearity (LIN) of spermatozoa after thawing. The supravital stain Hoechst 33258 and a fluoresceinated pea lectin were used to determine the $\%$ of viable spermatozoa with intact acrosomes. TSM and TEST were superior to EYC in terms of \%M and of \% viable, acrosome-intact spermatozoa. TSM and TEST produced equivalent VCL and LIN values, while EYC had clearly reduced VCL and LIN. There were no interactions between diluent and glycerol level. The 3\% glycerol level gave superior results to $5 \%$ glycerol for $\% \mathrm{M}$. EYC, which is widely used for cryopreservation of human spermatozoa, was not suitable for cynomolgus monkey semen. Artificial insemination with semen cryopreserved in TSM resulted in a healthy, full-term infant.
\end{abstract}

Key'words: cryopreservation; spermatozoa; acrosome; motility; macaques

\section{Introduction}

There are several practical reasons to develop cryopreservation methods for non-human primate spermatozoa, including the need to increase efficiency of simian breeding colonies and the advantage of avoiding the need for natural mating of exotic species in captivity. In addition, cryopreservation of macaque spermatozoa may provide a useful model system for investigating the nature and consequences of cryodamage to human spermatozoa. The cynomolgus monkey (Macaca fascicularis), whose male and female counterparts are reproductively similar to humans (Jaszczak \& Hafez, 1973; Overstreet \& VandeVoort, 1990), is easily maintained in captivity and can serve as a model species.

There have been only a few reports of conception in non-human primates after insemination with cryopreserved spermatozoa and none of these pregnancies resulted in an offspring (Leverage et al., 1972; Cho et al., 1975). The reasons for poor semen quality after cryopreservation in nonhuman primates are unknown and there is a lack of information on the characteristics of cryodamage in these species. In an effort to improve methodology for cryopreservation and to gain information on the nature of cryodamage to non-human primate spermatozoa, we utilized cryopreservation techniques similar to the methods employed for human spermatozoa in the largest

\footnotetext{
*Reprint requests to: E. Z. Drobnis, Reproductive Biology and Medicine, Suber House, University of California,
} Davis, CA 95616, USA. 
clinical study published to date (David et al., 1980). A TEST-yolk diluent was also evaluated, since these diluents are successful for a wide variety of species and studies have suggested that TEST may be superior to egg yolk-citrate for cryopreservation of human spermatozoa (Graham \& Crabo, 1978; Prins \& Weidel, 1986). An attempt was made to develop simple cryopreservation methods suitable for reproductive management of non-human primates.

\section{Materials and Methods}

Preparation of diluents. Three egg yolk-based extenders, buffered with sodium citrate (EYC), TES-Tris (TEST), or TES-Tris with skim milk (TSM) were prepared simultaneously from the same batch of freshly laid, untreated chicken eggs. EYC was prepared by diluting $2.9 \mathrm{~g}$ sodium citrate (Sigma Chemical Co., St. Louis, MO, USA), $1.0 \mathrm{~g}$ dextrose (Sigma), 0.015 g penicillin G (Sigma), and $0.025 \mathrm{~g}$ streptomycin sulphate (Sigma) to $100 \mathrm{ml}$ with h.p.l.c. grade water (Fisher Scientific, Pittsburgh, PA, USA). The TEST buffer solution (4.325 g TES, $1.0269 \mathrm{~g}$ TRIS in $100 \mathrm{ml}$ h.p.l.c. water) was modified from that described by Bolanos et al. (1983) by addition of the same concentrations of antibiotics and dextrose as in EYC. Egg yolk was added to the buffers to a concentration of $30 \%(\mathrm{v} / \mathrm{v})$. TSM buffer was prepared by adding skim milk (commercial fluid skim milk) to the TEST buffer to produce a $20 \%$ skim milk solution. All diluents were mixed vigorously, heated gradually to $60^{\circ} \mathrm{C}$, cooled to room temperature, and centrifuged at $800 \mathrm{~g}$ for $3 \mathrm{~h}$ to sediment yolk granules which would otherwise interfere with sperm analyses. Osmolality was approximately $320 \mathrm{mmol} / \mathrm{kg}$ for each diluent. The diluents were stored at $-25^{\circ} \mathrm{C}$ in $3-5-\mathrm{ml}$ samples until needed (Barkay \& Zuckerman, 1983), and were used within 15 days of preparation. Before an experiment, diluents were thawed in 50 C water and equilibrated to room temperature. The $\mathrm{pH}$ of each diluent was adjusted to 7.35-7.40.

Semen collection and processing. Six adult male cynomolgus monkeys were individually caged at the California Primate Research Center (CPRC) with lights on from 06:00 to $18: 00 \mathrm{~h}$ at $25-27^{\circ} \mathrm{C}$. The males were not preselected on the basis of semen quality or cryosurvival of spermatozoa. Monkeys were fed a diet of Purina monkey chow and water ad libitum. The males were trained to chair restraint and were electroejaculated with a Grass 6 stimulator equipped with EKG pad electrodes for direct penile stimulation $(30-50 \mathrm{~V}, 20 \mathrm{msec}$ duration, $18 \mathrm{pulses} / \mathrm{sec})$. A total of 12 ejaculates were studied (1-3 ejaculates per male). The mean ( \pm s.e.m.) volume of ejaculates, not including coagulum, was $285 \pm 22 \mu \mathrm{l}$ and the mean total number of spermatozoa was $467 \pm 92 \times 10^{6}$ cells. Each ejaculate was divided into 6 samples: 2 were diluted with TSM, two with TEST, and two with EYC to a concentration of $120 \times 10^{6}$ spermatozoa $/ \mathrm{ml}$. The two samples of each diluent pair were further diluted with glycerolated diluent which was added in thirds at $10-\mathrm{min}$ intervals to produce a final concentration of $60 \times 10^{6}$ spermatozoa/ml. This large dilution factor was necessary to allow post-thaw computer-assisted sperm analyses. The final concentrations of glycerol in the paired samples were 3 and $5 \%(\mathrm{v} / \mathrm{v})$. Dilution factors for semen ranged from $5: 1$ to $55: 1$ with a mean of $26: 1$. The cooling and freezing rates of spermatozoa were adapted from those reported by Graham \& Crabo (1978) which suggest that slow cooling rates combined with fast freezing rates result in highest post-thaw human sperm motility. To eliminate the requirement for a $5^{\circ} \mathrm{C}$, temperature-controlled room, the extended semen was packaged before cooling. Semen was drawn into $0.5 \mathrm{ml}$ wick and powder cryostraws and sealed with plastic beads (Continental Plastics, Delaven, WI, USA). Straws were sealed in a plastic bag to prevent accumulation of moisture on the outer surfaces, submerged in a $400 \mathrm{ml}$ beaker with room temperature water, and placed in a refrigerator. Over the next 120 min the water-bath temperature dropped to $5^{\circ} \mathrm{C}$. Straws were removed from the plastic bag and were manually lowered into the vapours of liquid nitrogen, using a rack equipped with thermocouple to standardize the freezing rate, which was maintained at $-60^{\circ} \mathrm{C} / \mathrm{min}$ until the rack was submerged in liquid nitrogen at $-196^{\circ} \mathrm{C}$. All samples were stored in liquid nitrogen for at least I week before evaluation.

Analysis of sperm motility and acrosomal integrity. Before freezing, a sample of each ejaculate was evaluated after dilution to $40-60 \times 10^{6}$ spermatozoa $/ \mathrm{ml}$ with glycerol-free TSM. Straws were thawed in a $37^{\circ} \mathrm{C}$ water bath for $5 \mathrm{~min}$. Fresh and thawed samples were assessed for sperm viability using the supravital dye Hoechst 33258 (H-258; Sigma) and for acrosomal integrity with fluorescein isothiocyanate-labelled Pisum sativum agglutinin (FITC-PSA; Vector Labs, Burlingame, CA, USA) (Cross et al., 1986). The method of Morales \& Cross (1989) for acrosomal staining of human spermatozoa on small-pore polycarbonate filters was adapted for application to non-human primate spermatozoa by extending the fixation time in paraformaldehyde to $12 \mathrm{~min}$ and decreasing the time of incubation with $\mathrm{H}-258$ to $8 \mathrm{~min}$. The filters were mounted on glass slides and 100 consecutive spermatozoa were scored on a blind basis with fluorescence microscopy for viability (exclusion of H258) and acrosomal status (Cross et al., 1986). Acrosome-reacted spermatozoa bound little or no FITC-PSA, while acrosome-intact spermatozoa bound label uniformly on the anterior head. Another group of spermatozoa exhibited patchy, irregular labelling in the acrosomal region and these cells were scored as having modified acrosomes.

An $8-\mu 1$ drop of pre-freeze or post-thaw semen, covered with a $22 \mathrm{~mm}$ square coverglass, was visualized with a $\times 20$ positive-phase objective and a condenser setting of 100 (pseudo-dark field) on an Olympus BH-series phasecontrast microscope (Scientific Instrument Co, Sunnyvale, CA, USA). An air curtain incubator (Sage Instruments, Model 279, Orion Research Inc, Cambridge, MA, USA) maintained the microscope stage at $37^{\circ} \mathrm{C}$. A model WV 3300 
colour Panasonic video camera with a $\times 2.5$ ocular lens was wired in series with an electronic field timer (For. A. Video Timer model VTG-33, Tokyo, Japan), a Panasonic model NV-8200 $\frac{1}{2}$ inch video cassette recorder, and a TV monitor. For each ejaculate, 3-5 fields were recorded, capturing at least 200 motile spermatozoa. Sperm motion characteristics were evaluated with Cell Soft Automated Semen Analyzer (Cryo Resources, Ltd, New York, NY, USA) using the parameter settings listed in Table 1. At least 100 spermatozoa per treatment were analysed for percentage motility $(\% \mathrm{M})$, curvilinear velocity (VCL), and linearity (LIN).

Table 1. Parameters used for computerized semen analysis

\begin{tabular}{lc}
\hline Parameter & Value \\
\hline Sampling frequency (frames/sec) & 30 \\
$\begin{array}{l}\text { No. of frames to analyse } \\
\text { Maximum allowable velocity }\end{array}$ & 10 \\
$\quad$ (microns/sec) & 250 \\
Minimum no. of points for motility & 1 \\
Minimum no. of points for velocity & 7 \\
Pixel scale ( $\mu$ m/pixel) & $0 \cdot 889$ \\
$\begin{array}{l}\text { Minimum size to exclude an object } \\
\text { from analysis (pixels) }\end{array}$ & 5 \\
$\begin{array}{l}\text { Maximum size to exclude an object } \\
\text { from analysis (pixels) }\end{array}$ & 35 \\
Threshold grey & $133-138$ \\
\hline
\end{tabular}

Artificial insemination. Semen preserved with TSM and 3\% glycerol was used for artificial insemination. The reproductive cycles of 3 female cynomolgus monkeys were monitored for signs of vaginal bleeding (onset of menses $=$ Day 1 of cycle). One insemination was performed during a single cycle for each female on Day 10 or 11 of the cycle. The cryopreserved spermatozoa were thawed and allowed to swim up into $0.5 \mathrm{ml}$ modified BWW Medium (Overstreet et al., 1980). Intrauterine insemination was performed using a 25 -gauge needle that was placed into the uterine lumen by ultrasound-guided, transabdominal puncture (Tarantal et al,, 1990). A total of $1 \times 10^{6}$ spermatozoa ( $90 \%$ motile) in $100 \mu l$ was injected per insemination.

Statistical analysis. The data were analysed in a $3 \times 2$ factorial design, blocked by ejaculate, with 3 diluents and 2 levels of glycerol. Differences amongst means were tested using Tukey's multiple range test (Snedecor \& Cochran, 1967). All data are expressed as mean \pm s.e.m.

\section{Results}

All parameters of motility were affected by cryopreservation, regardless of the treatment (Table 2). However, there was no correlation $(R=0.55)$ between the extent of semen dilution and post-thaw survival of spermatozoa. The results for diluents and glycerol treatments were independent. In general, the $\% \mathrm{M}, \mathrm{VCL}$ and LIN were all reduced significantly in comparison with the values for prefreeze extended semen. The data on the effects of amount of glycerol are given separately in Table 3. The large difference in means of post-thaw sperm motility characteristics in EYC-treated samples masked differences due to glycerol level in the other two diluents. When the analysis was repeated without EYC treatment values, more accurate assessments of glycerol effects were obtained for spermatozoa preserved in TEST and TSM. As shown in Table 3, \% M and VCL were higher following treatments with $3 \%$ glycerol than with $5 \%$ glycerol $(P=0.0239$ and 0.0043 respectively). Glycerol level did not significantly affect LIN. For media containing 3\% glycerol, the $\% \mathrm{M}$ was highest for semen preserved in TSM followed by TEST and EYC, with values ranging from 59 to $82 \%, 47$ to $73 \%$ and 1 to $24 \%$, respectively (data not shown). For media containing $3 \%$ glycerol, post-thaw LIN was also higher for spermatozoa in TSM $(5 \cdot 3 \pm 0 \cdot 24)$ followed by TEST $(4.8 \pm 0.24)$ and EYC $(3.4 \pm 0 \cdot 25, P=0 \cdot 187$, data not shown). VCL was greater in TSM and TEST than in EYC regardless of the amount of glycerol (Table 2). 
Table 2. Post-thaw sperm motility after cryopreservation of cynomolgus spermatozoa in three different diluents

\begin{tabular}{lccc}
\hline Treatment & $\begin{array}{c}\text { Motility } \\
(\%)\end{array}$ & $\begin{array}{c}\text { Curvilinear } \\
\text { velocity } \\
(\mu \mathrm{m} / \mathrm{sec})\end{array}$ & Linearity \\
\hline Fresh* & $91 \pm 0 \cdot 8$ & $113 \pm 1$ & $8 \cdot 0 \pm 0 \cdot 3$ \\
EYC & $12 \pm 2^{\mathrm{a}}$ & $46 \pm 2^{\mathrm{a}}$ & $3 \cdot 5 \pm 0 \cdot 2^{\mathrm{a}}$ \\
TEST & $56 \pm 3^{\mathrm{b}}$ & $63 \pm 3^{\mathrm{b}}$ & $4 \cdot 7 \pm 0 \cdot 2^{\mathrm{b}}$ \\
TSM & $67 \pm 2^{\mathrm{c}}$ & $60 \pm 3^{\mathrm{b}}$ & $5 \cdot 2 \pm 0 \cdot 2^{\mathrm{b}}$ \\
\hline
\end{tabular}

Values are mean \pm s.e.m. for 12 ejaculates.

*Sperm motility before freezing in semen diluted with TSM.

Within columns, values with different superscripts differ significantly $(P<0.05)$.

Table 3. Post-thaw sperm motility after cryopreservation of cynomolgus spermatozoa in extenders with $3 \%$ glycerol or $5 \%$ glycerol

\begin{tabular}{lccc}
\hline Glycerol & $\begin{array}{c}\text { Motility } \\
(\%)\end{array}$ & $\begin{array}{c}\text { Curvilinear } \\
\text { velocity } \\
(\mu \mathrm{m} / \mathrm{sec})\end{array}$ & Linearity \\
\hline $3 \%$ & $65 \pm 2^{\mathrm{a}}$ & $64 \pm 3^{\mathrm{a}}$ & $5 \cdot 0 \pm 0 \cdot 2^{\mathrm{a}}$ \\
$5 \%$ & $58 \pm 3^{\mathrm{b}}$ & $58 \pm 3^{\mathrm{b}}$ & $4 \cdot 8 \pm 0 \cdot 2^{\mathrm{a}}$ \\
\hline
\end{tabular}

Values are mean \pm s.e.m. for 12 ejaculates frozen in TEST or TSM diluents.

Within columns, values with different superscripts differ significantly $(P<0.05)$.

The viability (dye exclusion) and acrosomal integrity of spermatozoa were significantly reduced in all treatment groups after cryopreservation (Table 4). Samples diluted with TSM and TEST had a larger percentage of viable cells with intact acrosomes after thawing than did samples diluted with EYC. There was no significant effect of glycerol level on acrosomal integrity. Unlike fresh semen, cryopreserved semen contained many spermatozoa with patchy, irregularly labelled acrosomes (modified acrosomes, Table 4), together with spermatozoa having evenly stained acrosomal contents (intact acrosomes, Table 4).

Of the 3 female monkeys that were inseminated with spermatozoa cryopreserved in TSM and 3\% glycerol, 1 became pregnant. Pregnancy was confirmed and monitored with ultrasound. A healthy, male infant was delivered by Caesarean section at 154 days of gestation.

\section{Discussion}

In this study we have evaluated several methods for cryopreservation of cynomolgus monkey semen. In general, all treatments significantly reduced the semen quality, but spermatozoa preserved in TEST-buffered diluents were superior to those preserved in EYC in terms of motility and acrosomal integrity. When semen preserved by TSM was used for artificial insemination, a pregnancy was obtained in 1 of 3 attempts. This is the first well-documented report of a non-human primate infant resulting from insemination with cryopreserved semen. 
Table 4. Acrosomal status of viable cynomolgus spermatozoa cryopreserved in TSM, TEST or EYC and effects of $3 \%$ or $5 \%$ glycerol in all diluents*

\begin{tabular}{ccccc}
\hline & & \multicolumn{3}{c}{$\%$ Viable } \\
\cline { 3 - 5 } & $\begin{array}{c}\% \\
\text { not viable }\end{array}$ & $\begin{array}{c}\text { Acrosome } \\
\text { intact }\end{array}$ & $\begin{array}{c}\text { Modified } \\
\text { acrosome }\end{array}$ & $\begin{array}{c}\text { Acrosome } \\
\text { reacted }\end{array}$ \\
\hline Diluent & & & & \\
EYC & $57 \pm 2^{\mathrm{a}}$ & $16 \pm 2^{\mathrm{a}}$ & $24 \pm 2^{\mathrm{a}}$ & $3 \cdot 7 \pm 1$ \\
TEST & $28 \pm 2^{\mathrm{b}}$ & $31 \pm 4^{\mathrm{b}}$ & $35 \pm 3^{\mathrm{b}}$ & $4 \cdot 4 \pm 1$ \\
TSM & $27 \pm 2^{\mathrm{b}}$ & $38 \pm 4^{\mathrm{b}}$ & $28 \pm 2^{\mathrm{a} \cdot \mathrm{b}}$ & $6 \cdot 7 \pm 1$ \\
Glycerol & & & & \\
$3 \%$ & $35 \pm 2$ & $31 \pm 3$ & $29 \pm 2$ & $4 \cdot 8 \pm 1$ \\
$5 \%$ & $40 \pm 2$ & $25 \pm 3$ & $29 \pm 2$ & $5 \cdot 1 \pm 1$ \\
\hline
\end{tabular}

*Values are mean \pm s.e.m. for 12 ejaculates.

Within columns, values with different superscripts differ significantly $(P<0.05)$.

Cynomolgus semen is highly concentrated and therefore large dilution rates were necessary to enable computer-assisted sperm motility analysis. It is possible that the dilution rate had either positive or negative effects on sperm characteristics which might not be consistent between diluents used in this study. Dilution effects are species specific (Watson, 1979) and no such effects have been reported for cryopreservation of non-human primate semen.

Although EYC is used extensively for the preservation of human semen (David et al., 1980; Foote, 1982; Barkay \& Zuckerman, 1983) and has been used for non-human primate semen (Leverage et al., 1972), this was the least effective cryopreservation diluent used in this study. Cryopreservation in EYC reduced \% $\%$, VCL, LIN and the percentage of cells with intact acrosomes to a greater degree than when other media were used. Some studies of human sperm cryopreservation have also reported sub-optimal results with EYC when compared to other diluents using various freezing techniques. With glycerol levels of $4 \%(G r a h a m \&$ Crabo, 1978) and $7.5 \%$ (Prins \& Weidel, 1986), cryopreservation in EYC resulted in average post-thaw motility of 12.4 and $49 \%$ respectively. Using different cryopreservation techniques, Leverage et al. (1972), obtained an average post-thaw motility of $34 \%$ when the semen of rhesus monkeys (Macaca mulatta) was cryopreserved with EYC. It is possible that the results of the present study show a species-specific effect and that spermatozoa from cynomolgus macaques are poorly preserved in EYC. Alternatively, good results with EYC diluent may require a specific combination of cryopreservation techniques not included in this study.

Glycerol is only effective as a cryoprotectant of cynomolgus monkey spermatozoa within a narrow range of concentrations. In this study, a $2 \%$ difference in glycerol level gave significant differences in post-thaw percentage motility, with $3 \%$ glycerol resulting in the highest sperm survival (Table 3). Previous reports have indicated that glycerol levels of 2.5 to $10 \%$ most effectively support survival of macaque spermatozoa, depending on the type of extender. However, glycerol levels of 10-20\% markedly reduced sperm motility after thawing (Cho \& Honjo, 1973; Mahone \& Dukelow, 1978).

Assessment of sperm motility characteristics revealed that velocities of spermatozoa in fresh, extended semen were nearly twice that of cryopreserved spermatozoa. Sperm trajectories were also considerably more linear in fresh, extended semen than in cryopreserved semen. These changes probably reflect cryodamage, and the extent of the change was dependent on the freezing treatment. Cryopreservation resulted in considerable acrosomal damage. Complete loss of acrosomes occurred with highest frequency in the EYC group, as reflected in the low percentage of viable spermatozoa with intact acrosomes (Table 4). TSM and TEST were more effective than EYC in 
preserving acrosomal integrity by many of the intact spermatozoa had patchy, non-uniform staining of the acrosomal region. This modified staining pattern was seldom seen in the fresh samples ( $<1 \%$, data not shown), and is probably a consequence of acrosomal damage or alteration during cryopreservation. Ultrastructural changes of the acrosome have been associated with freeze preservation and thawing of human spermatozoa (Alexander, 1977; Mahadevan \& Trounson, 1984; Heath et al., 1985). Electron microscopy studies of frozen-thawed human and macaque spermatozoa have revealed acrosomal swelling, various degrees of disintegration of the plasmalemma and outer acrosomal membrane, and loss or leakage of acrosomal material (Leverage et al., 1972). Spermatozoa with degenerative acrosomes exhibit faint, or pale-irregular staining of the anterior portion of the head, giving the acrosome a ruffled appearance at the light microscopic level (Oettle \& Soley, 1986). Similar manifestations of cryodamage are apparently revealed by the modified acrosomal staining patterns observed in spermatozoa labelled with FITC-PSA.

This work was supported in part by NIH grants HD15149, HD25907 and RR00169.

\section{References}

Alexander, N.J. (1977) Surface structure of spermatozoa frozen for artificial insemination. Andrologia 9, 155162

Barkay, J. \& Zuckerman, H. (1983) Cryopreservation and pooling of spermatozoa. In Treatment of Male Infertility IV, pp. 263-281. Eds J. Bain, W. Schill \& L. Schwarzstein. Springer-Verlag, New York.

Bolanos, J.R., Overstreet, J.W. \& Katz, D.F. (1983) Human sperm penetration of zona-free hamster eggs after storage of the semen for 48 hours at $2^{\circ} \mathrm{C}$ to $5^{\circ} \mathrm{C}$. Fert. Steril. 39, 536-541.

Cho, F. \& Honjo, S. (1973) A simplified method for collecting and preserving cynomolgus macaque semen. Japan. J. med. Sci. Biol. 26, 261-268.

Cho, H., Honjo, S. \& Makita, T. (1975) Fertility of frozen-preserved spermatozoa of cynomolgus monkeys. In Contemporary Primatology, pp. 125-133. Eds S. Kondo, M. Kawai \& A. Ehara. S. Karger, Basel.

Cross, N.L., Morales, P., Overstreet, J.W., \& Hansen, F.W. (1986) Two simple methods for detecting acrosome-reacted human sperm. Gamete Res. 15, 213-226.

David, G., Czyglik, F., Mayaux, M.J., Martin-Boyce, A. \& Schwartz, D. (1980) Artificial insemination with frozen sperm: protocol, method of analysis and results for 1188 women. Br. J. Obstet. Gynaecol. 87, 1022-1028.

Foote, R.H. (1982) Cryopreservation of spermatozoa and artificial insemination: Past, present, and future. J. Androl. 3, 85-100.

Graham, E.F. \& Crabo, B.G. (1978) Some methods of freezing and evaluating human spermatozoa. In The Integrity of Frozen Spermatozoa, pp. 274-304. National Academy of Science, Washington DC.

Heath, E., Jeyendran, R.S., Perez-Pelaez, M. \& Sobrero, A.J. (1985) Ultrastructural categorization of human sperm cryopreserved in glycerol and in TESTCY. Int . J. Androl. 8, 101-110.

Jaszczak, S. \& Hafez, E.S.E. (1973) Sperm migration through the uterine cervix in the macaque during the menstrual cycle. Am. J. Obstet. Gynecol. 115, 10701082.

Leverage, W.E., Valerio, D.A., Schultz, A.P., Kingsbury, E. \& Dorey, C. (1972) Comparative study on the freeze preservation of spermatozoa: primate, bovine, and human. Lab. Anim. Sci. 6, 882-889.

Mahadevan, M.M. \& Trounson, A.O. (1984) Relationship of fine structure of sperm head to fertility of frozen human semen. Fert. Steril. 41, 287-293.

Mahone, J.P. \& Dukelow, W.R. (1978) Semen preservation in Macaca fascicularis. Lab. Anim. Sci. 28, 556-561.

Morales, P. \& Cross, N.L. (1989) A new procedure for determining acrosome status of very small numbers of human sperm. J. Histochem. Cytochem. 37, 12911292.

Oettlé, E.E. \& Soley, J.T. ([986) Ultrastructural changes in the acrosome of human sperm during freezing and thawing: a pilot trial. Arch. Androl. 17, 145-150.

Overstreet, J.O. \& VandeVoort, C.A. (1990) Sperm transport in the female genital tract. In Gamete Physiology. Ed. R. H. Asch. Raven Press, New York (in press)

Overstreet, J.W., Yanagimachi, R., Katz, D.F., Hayashi, K. \& Hanson, F.W. (1980) Penetration of human spermatozoa into the human zona pellucida and the zona-free hamster egg: a study of fertile donors and infertile patients. Fert. Steril. 33, 534-542.

Prins, G.S. \& Weidel, L. (1986) A comparative study of buffer systems as cryoprotectants for human spermatozoa. Fert. Steril. 46, 147-149.

Snedecor, G.W. \& Cochran, W.G. (1967) Statistical Methods, 6th edn, p. 272. Iowa State University Press, Ames.

Tarantal, A.F., VandeVoort, C.A. \& Overstreet, J.W. (1990) Intrauterine insemination with uitrasound guidance in the Cynomolgus monkey (Macaca fascicularis). J. med. Primatol. (in press).

Watson, P.F. (1979) The preservation of semen in mammals. Oxford Rev. Reprod. Biol. 1, 283-350.

Received 21 November 1989 